\section{P29 DOES CHRONIC SLEEP DEPRIVATION INFLUENCE THE RISK OF DEPRESSION IN YOUNG PEOPLE? A LONGITUDINAL AND GENDER-BASED ANALYSIS OF THE BASUS COHORT STUDY}

$1,2 \mathrm{Al}$ Conklin* ${ }^{2} \mathrm{CA}$ Yao ${ }^{2,3} \mathrm{CG}$ Richardson. ${ }^{1}$ Faculty of Pharmaceutical Sciences, University of British Columbia, Vancouver, Canada; ${ }^{2}$ Centre for Health Evaluation and Outcome Sciences, Providence Healthcare Research Institute, Vancouver, Canada; ${ }^{3}$ School of Population and Public Health, University of British Columbia, Vancouver, Canada

\subsection{6/jech-2018-SSMabstracts. 155}

Background Chronic exposure to sleep deprivation may increase risk of depression in young people who are particularly vulnerable to changes in sleep and mental health. Sleep deprivation and incident depression may also differ by gender. We investigated the prospective association between cumulative sleep deprivation and subsequent levels of depressive symptomatology among adolescents from a gender perspective.

Methods A longitudinal study of 3071 young people in the British Columbia Adolescent Substance Use Survey (BASUS) cohort with three sleep time and two depression measures (CESD) over 12 months (2011-12). Multivariable linear regression models with sex interaction terms estimated genderspecific associations between self-reported chronic sleep deprivation and changes in depressive symptomatology; post-estimation analysis calculated adjusted mean depression scores for each level of cumulative sleep deprivation.

Results Cumulative sleep deprivation was associated with a monotonic increase in depression scores at follow-up in young women, but no consistent pattern was seen in young men. During follow-up, $15 \%$ of young women were chronically sleep deprived and 29\% were depressed (CESD $\geq 24$ ). Young women reporting chronic exposure to sleep deprivation had higher CESD scores at follow-up (21.50 points, CI95 19.5523.45), than those reporting no history (16.59 (CI95 15.7217.45); associations remained after multivariable adjustment (19.48, CI95 17.59-21.38).

Conclusion Results suggested that chronic sleep deprivation increases the risk of major depression among young women. Mental health promotion for young people should include relevant strategies to ensure young women can achieve recommended amounts of sleep.

\section{P30 RAPID OVERVIEWS FOR PRAGMATIC TRANSFER OF KNOWLEDGE FROM RESEARCH TO PRACTICE}

${ }^{1} \mathrm{~L}$ Long*, ${ }^{2} \mathrm{VL}$ Joshi, ${ }^{2} \mathrm{LH}$ Tang, ${ }^{1} \mathrm{RS}$ Taylor, ${ }^{2} \mathrm{AD}$ Zwisler. ${ }^{1}$ University of Exeter Medical School, Institute of Health, Exeter, UK; ${ }^{2}$ Danish Knowledge Centre for Rehabilitation and Palliative Care (RHEPA), University of Southern Denmark, Nyborg, Denmark

\subsection{6/jech-2018-SSMabstracts.156}

Background Today, many more people are surviving sudden cardiovascular events and living longer with cardiovascular disease, thanks to advances in preventive strategies and advanced treatment. Hence, rehabilitation and palliative care interventions have become an important focus for improving the lives of patients living with cardiovascular diseases.

Drawing on 'review of reviews' and rapid review methodology, as well as evidence gap maps, we worked with non-academic stakeholders at the Danish Heart Foundation (DHF) to co-produce a contemporary overview to uniquely bring together the evidence for the efficacy of rehabilitation and palliative care across a wide spectrum of cardiovascular diseases. The overview summarises the current evidence but also identifies where gaps in the evidence exist.

Methods End user feedback was sought on the protocol and at key points in the overview process. Six bibliographic databases were searched for systematic reviews. Only articles published after 2009 were included. Quality of SRs was assessed using the R-AMSTAR tool. A narrative synthesis was performed and a summary of the most recent systematic review (s)/meta-analyses for efficacy of rehabilitation for each of the included CVD conditions presented using accessible graphic visualisations of data (infographics).

Results The overview finds that the evidence for rehabilitation in coronary heart disease and heart failure is well established as well as supervised exercise training in intermittent claudication. Improved access to rehabilitation is needed for these indications. There is a dearth of studies on the efficacy of dietary and smoking interventions in CVD indications. Also, further research is needed on palliative care interventions for heart failure and other life-threatening cardiovascular diseases. Conclusion A pragmatic, flexible approach to conducting this overview, together with meaningful involvement of end users, facilitated completion of a comprehensive overview to deadline (six months), identified gaps in the evidence, improved accessibility of findings and ensured relevance to real world settings. Limitations included no attempt to search for individual randomised controlled trials (RCTs), to perform updates or undertake de novo SRs. We only focused on the effectiveness of rehabilitation and palliative interventions for CVD relative to control and did not consider the relative effectiveness of different components of interventions (for example, type, duration, frequency of exercise training) or settings.

The overview highlights the need to prioritise high quality studies that establish the effectiveness of rehabilitation in cardiovascular indications where there is currently little or no evidence.

\section{P31 ENGAGING WITH STAKEHOLDERS TO INFORM THE DEVELOPMENT OF A COMPUTER MODEL FOR THE NHS HEALTH CHECK PROGRAMME: WORKHORSE PROJECT}

${ }^{1}$ L Hyseni*, ${ }^{1} \mathrm{M}$ Guzman-Castillo, ${ }^{1} \mathrm{C}$ Kypridemos, ${ }^{1} \mathrm{~B}$ Collins, ${ }^{1} \mathrm{E}$ Schwaller, ${ }^{1} \mathrm{C}$ Capewell, ${ }^{2} \mathrm{~A}$ Boland, ${ }^{2} \mathrm{R}$ Dickson, ${ }^{1} \mathrm{M}$ O'Flaherty, ${ }^{1} \mathrm{~F}$ Lloyd-Williams. 'Public Health and Policy, University of Liverpool, Liverpool, UK; ${ }^{2}$ Health Services Research, University of Liverpool, Liverpool, UK

\subsection{6/jech-2018-SSMabstracts. 157}

Background The NHS Health Check Programme (NHSHCP) is a multifactorial 'risk-reduction' programme offered to all adults in England aged 40-74. Studies of the NHSHCP suggest that it might be further improved by including additional conditions and by facilitating local commissioning. This project aims to use stakeholder engagement to co-produce and develop a validated open-source/open access, flexible decision support tool to enable local commissioners to quantify the local effectiveness, cost-effectiveness and equity of the NHSHCP.

Methods We have adopted the innovative approach of engaging with key stakeholders in four iterative workshops, to coproduce model and scenario specifications. We identified stakeholders using our extensive networks and using the snowballing techniques. In workshop 1 , we used the validated Hovmand 'group model building' approach to engage stakeholders in a series of pre-piloted, structured, small group 